\title{
Determination of EOP from VLBI in IAA
}

\author{
E. Skurihkina \\ Institute of Applied Astronomy of Russian Academy of Science
}

\begin{abstract}
Results of Earth orientation parameter (EOP) determinations from NEOS-A and CORE programs reduced using the package OCCAM version 3.5 at the Institute of Applied Astronomy of Russian Academy of Science (IAA) are presented. Results of EOP determination using different reference stations, relativistic models, the IERS Standards (1992) and IERS Conventions (1996) are also presented.
\end{abstract}

\section{Introduction}

The determination of EOPs at IAA is based on the use of new methods of space geodesy (SLR, GPS, VLBI). VLBI is the only method that determines all EOP - $\mathrm{x}$ - and $\mathrm{y}$ - pole-coordinates, Universal Time, corrections to nutation angles - dPsi and dEps. Station positions are also determined for all observational sessions.

OCCAM is used to process VLBI observational data. It was built by Nestor Zarraoa for geodetic tasks. At the present time Titov (version 3.4 (Titov and Zarraoa, 1997)) is the coordinator of OCCAM. Version 3.5 is used at IAA. It is a version improved in the method of reduction, the size of processing information (maximum size of some values, such as the number of stations, the number of baselines, the number of scans and so on). The results of this work is a significant reduction of random and systematic errors of the EOP estimations.

The reduction part of OCCAM is based on IERS Conventions (1996). The one exception is the relativistic model, which uses IERS Standards (1992) because of ambiguity in the IERS Conventions (1996). A Kalman Filter (Titov 1996) is used to estimate the stochastic parameters such as tropospheric delay in the zenith and clock by a random walk model, and EOP and station positions as constants for each session. There is no provision for source position improvement in this version of OCCAM.

\section{EOP Service in IAA}

EOP and station positions are determined from NEOS-A VLBI observations in the EOP Service of IAA. The EOP Service automatically obtains observational data (Voinov 1997), converts them to NGS format (Surkis 1997), processes them and sends results to centers processing EOP - the Institute of Metrology of Time and Space in Russia; the IERS Central Bureau and to the IERS Subbureau for Rapid Service and Prediction. The last global solution was obtained 
using the ITRF97 catalog of stations positions and the radio source position catalog RSC(IAA) 99R02 of G. A. Krasinsky (Feb. 1998). It was done by processing 304 sessions of NEOS-A from May 1993. The result is the column EOP(IAA)98R01. (rms from EOP $(\mathrm{CO} 4)$ seen in Table 1 that also presents the 1996 , and 1997 global solutions of IAA).

Table 1. Rms EOP(IAA)96R01, 97R01, 98R01 from EOP(IERS)C04 (beginning May 1993).

\begin{tabular}{lccc}
\hline \hline & 1996 & 1997 & 1998 \\
\hline Xp, mas & 0.30 & 0.37 & 0.25 \\
Yp, mas & 0.26 & 0.26 & 0.21 \\
UT1-UTC, 0.1 ms & 0.19 & 0.15 & 0.12 \\
dPsi, mas & 0.39 & 0.38 & 0.37 \\
dEps, mas & 0.19 & 0.16 & 0.15 \\
\hline
\end{tabular}

IAA also processed IRIS-A observations beginning in 1984. Wettzell is the reference station when we used NEOS-A observations, and WESTFORD is the reference station when IRIS-A observations were used. The results of IAA EOP determination are presented in Figure 1.

\section{Comparison of EOP Estimations Obtained from NEOS-A and CORE-A}

IAA has obtained EOP and station positions from processing VLBI observations of the CORE-A and CORE-B programs. The rms of EOP estimations obtained from processing these observations from 1997 to 1999 from IERS EOP(C04) are presented in Table 2. These values may be used as the accuracy of EOP time series. The first column shows $0.5(\mathrm{Xp}+\mathrm{Yp})$, where $\mathrm{Xp}$ is the $\mathrm{x}$-position of the pole, $\mathrm{Yp}$ is the y position of the pole (in mas). The second column shows Universal Time (in $0.1 \mathrm{~ms}$ ), and the third column shows value $(\sigma(\delta \psi) \cdot \sin \epsilon+$ $\sigma(\delta \epsilon)) / 2$ for the nutation angles.

It seems that the accuracy of EOP obtained from the CORE-A and NEOSA programs is the best and for residuals of nutation angles NEOS-A is better. The accuracy of EOP obtained for CORE-B is worse then for CORE-A, and NEOS-A is better for all kinds of EOP.

The rms for the differences of EOP obtained from NEOS-A and COREA programs for the raw differences (without correction for bias or trend) and corrected for bias or trend differences is presented in the top two rows in Table 3 and the third row shows the maximum deviation of EOP.

\section{Testing of EOP with Different Reference Stations}

We chose sessions of the NEOS-A program when three stations - WETTZELL, GILCREEK and NRAO20 - observed simultaneously to research the effect of different reference stations. Each of these stations were used as reference station 
IRIS-A (+), NEOS-A $(\diamond):$ Xp

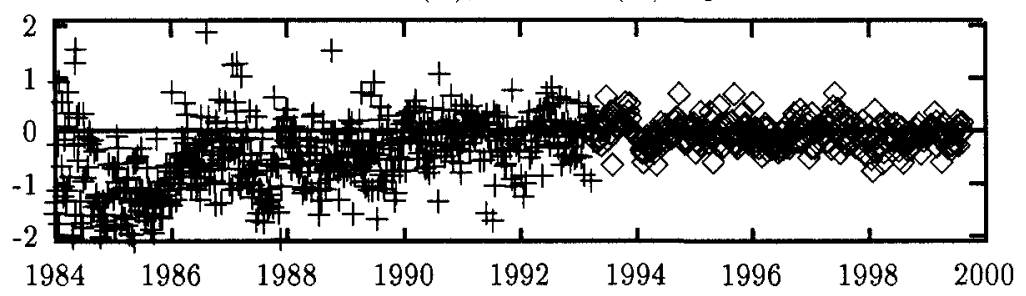

IRIS-A $(+)$, NEOS-A $(\diamond):$ Yp

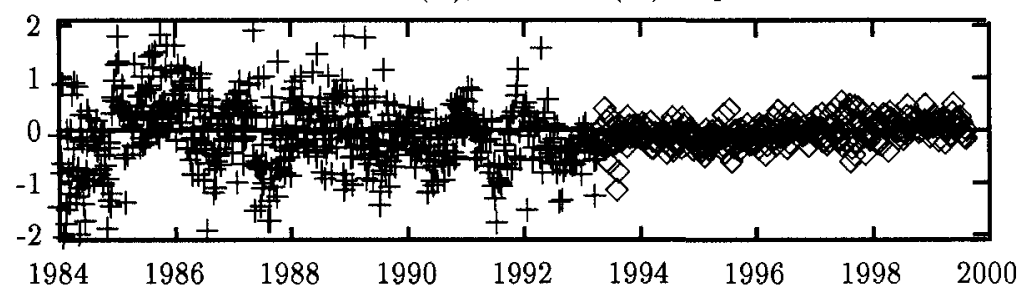

IRIS-A (+), NEOS-A $(\diamond):$ UT1-UTC

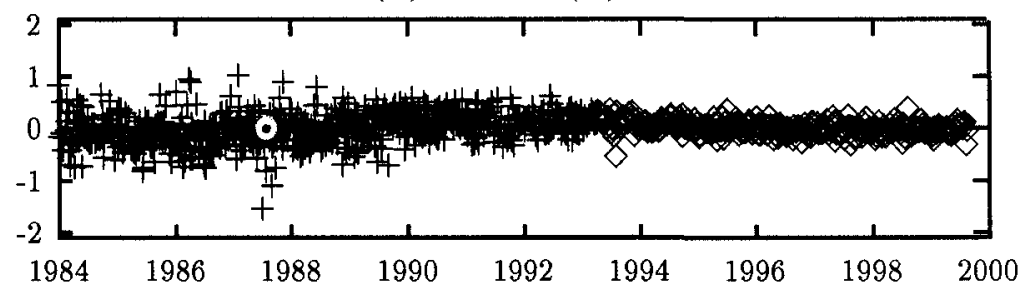

IRIS-A (+), NEOS-A $(\diamond):$ dPsi

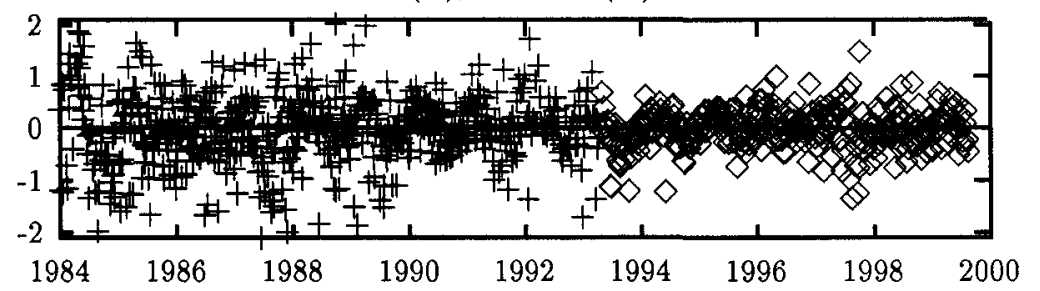

IRIS-A (+), NEOS-A ( $\diamond)$ : dEps

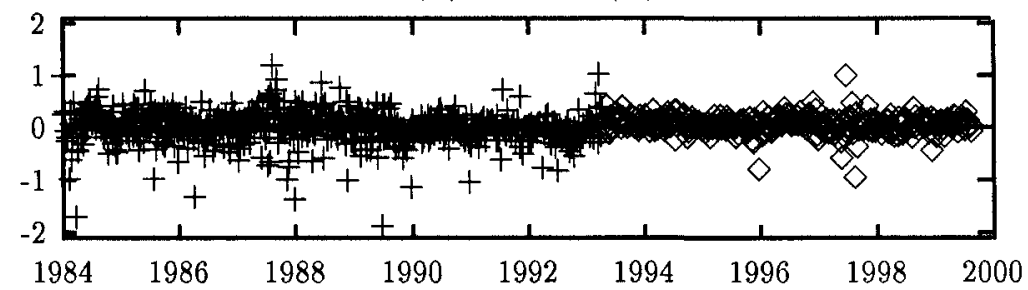

Figure 1. EOP estimations of OCCAM from EOP(IERS)C04. 
Table 2. rms of EOP estimations obtained from NEOS-A, CORE-A, CORE-B from EOP(IERS)C04 from 1997-1999

\begin{tabular}{lccc}
\hline \hline & $\begin{array}{c}\text { Pole } \\
\text { mas }\end{array}$ & $\begin{array}{c}\text { UT1-UTC } \\
0.1 \mathrm{~ms}\end{array}$ & $\begin{array}{c}\text { Nut } \\
\text { mas }\end{array}$ \\
\hline CORE-A & 0.22 & 0.11 & 0.22 \\
CORE-B & 0.32 & 0.18 & 0.42 \\
NEOS-A & 0.22 & 0.11 & 0.30 \\
\hline
\end{tabular}

for each of these sessions. Then the EOP estimates were compared. Table 3 presents the rms corrected for bias and trend differences and the maximal deviation of

- 1) EOP estimations obtained from NEOS-A and CORE-A;

- 2) EOP estimations obtained from NEOS-A with reference stations

- a. GILCREEK and WETTZELL

- b. NRAO20 and GILCREEK

- c. NRAO20 and WETTZELL;

- 3) EOP estimations from NEOS-A program with WETTZELL as reference station and with the relativistic model of IERS Standards 92 and the relativistic model of the IERS Conventions 96.

From the middle part of Table 3 we can see that the change of reference station has more influence on pole position (Xp, $\mathrm{Yp}$ ) and corrections for nutation angles, particularly for $\delta \psi$ between NRAO20 and WETTZELL. It will be noted that the appropriate values in the case of the comparison between NEOS-A and CORE-A results (for $\mathrm{Xp}, \mathrm{Yp}, \delta \psi, \delta \epsilon$ ) are three to four times larger then the ones for different reference stations. Results for UT1-UTC in both cases are similar.

For separate sessions EOP deviations are very large. For UT1 it amounts to about $5 \%$, for $\mathrm{Xp}, \mathrm{Yp}-$ about $10 \%$, for nutation angles - more then $20 \%$.

\section{Comparison of EOP obtained using the relativistic model of the IERS Standards (1992) and IERS Conventions (1996)}

For this comparison NEOS-A observations were used, with WETTZELL as the reference station. The rms for the differences (shown in Table 3 ) were for $\mathrm{Xp}$, $\mathrm{Yp},(\delta \psi) \cdot \sin \epsilon, \delta \epsilon$ smaller then 0.02 mas, and for UT1-UTC - smaller than $0.01 \mathrm{msec}$. The maximum differences were for $\mathrm{Xp}, \mathrm{Yp},(\delta \psi) \cdot \sin \epsilon, \delta \epsilon$ smaller than 0.2 mas, and for U'T1-UTC - smaller then 0.05 mas. These are about five times smaller than for the differences obtained using different reference stations. 
Table 3. rms and maximum differences between EOP estimations

\begin{tabular}{lccccc}
\hline \hline & $\begin{array}{c}\mathrm{dXp} \\
\text { mas }\end{array}$ & $\begin{array}{c}\mathrm{dYp} \\
\text { mas }\end{array}$ & $\begin{array}{c}\mathrm{dUT} \\
0.1 \mathrm{~ms}\end{array}$ & $\begin{array}{c}\mathrm{dPsi}{ }^{*} \sin \epsilon \\
\text { mas }\end{array}$ & $\begin{array}{c}\mathrm{dEps} \\
\text { mas }\end{array}$ \\
\hline NEOS - CORE-A & & & & & \\
rms(raw dif.) & 0.36 & 0.26 & 0.16 & 0.16 & 0.23 \\
rms(corr. dif.) & 0.33 & 0.25 & 0.14 & 0.16 & 0.23 \\
max abs & -1.08 & -0.86 & -0.46 & 0.46 & -0.82 \\
\hline GILC - WETT & & & & & \\
rms(raw dif.) & 0.08 & 0.07 & 0.04 & 0.09 & 0.09 \\
rms(corr. dif.) & 0.08 & 0.06 & 0.04 & 0.09 & 0.09 \\
max abs & -0.45 & -0.34 & 0.18 & 0.35 & 0.32 \\
NR20 - GILC & & & & & \\
rms(raw dif.) & 0.10 & 0.07 & 0.04 & 0.07 & 0.05 \\
rms(corr. dif.) & 0.10 & 0.07 & 0.03 & 0.07 & 0.05 \\
max abs & 0.78 & -0.38 & -0.19 & -0.46 & -0.19 \\
NR20 - WETT & & & & & \\
rms(raw dif.) & 0.12 & 0.05 & 0.03 & 0.11 & 0.08 \\
rms(corr. dif.) & 0.11 & 0.05 & 0.03 & 0.11 & 0.08 \\
max abs & 0.75 & -0.28 & 0.15 & -0.55 & 0.30 \\
\hline rel IS92- IC96 & & & & & \\
rms(raw dif.) & 0.02 & 0.02 & 0.01 & 0.02 & 0.01 \\
rms(corr. dif.) & 0.02 & 0.02 & 0.01 & 0.02 & 0.01 \\
max abs & -0.09 & -0.11 & -0.03 & 0.14 & 0.07 \\
\hline
\end{tabular}

\section{References}

Surkis I.F. MARK-3 File Format and Systems to Decode It. Comm. IAA, 1997, No 104 - in Russian.

Titov O.A. Use of Least Square Collocation Method for processing of VLBI Observations. PhD Thesis, St.Petersburg, IAA, 1996, 50-61 - in Russian.

Titov O., Zarraoa N. OCCAM 3.4 User's Guide. Comm. IAA, 1997, No 69.

Voinov A.V. Specialized Software Package for the Data Transfer via Internet in the Framework of the EOP Service of IAA RAS. Trans. IAA, 1997, 145-154 - in Russian. 